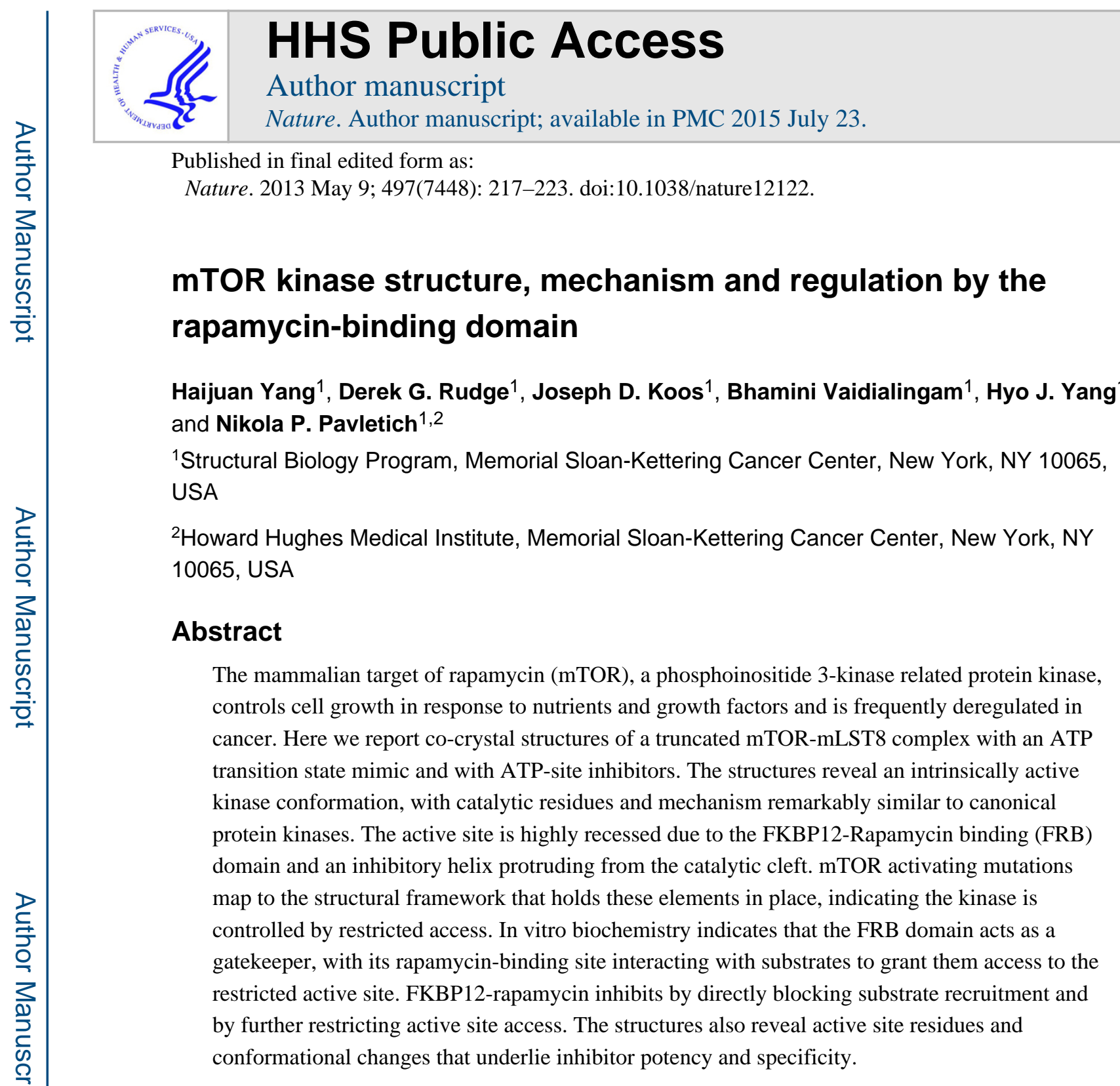

The mTOR pathway controls cell growth in response to energy, nutrients, growth factors and other environmental cues, and it figures prominently in cancer ${ }^{1,2}$. Central to the pathway is the mammalian target of rapamycin (mTOR) protein that belongs to the phosphoinositide 3-kinase (PI3K)-related protein kinase (PIKK) family ${ }^{3}$. mTOR assembles into two complexes with distinct inputs and downstream effects. mTOR Complex 1 (mTORC1) is defined by its RAPTOR subunit ${ }^{4-6}$, which is replaced by RICTOR in TTORC2 $^{6,7}$. Both

Reprints and permissions information is available at www.nature.com/reprints.

Correspondence and requests for materials should be addressed to pavletin@mskcc.org.

Author Contributions H.Y. and N.P.P. designed the experiments, solved the structures and wrote the manuscript. H.Y., D.G.R., J.D.K., B.M. and H.J.Y. carried out protein production, crystallization, and biochemical experiments. H.Y. performed all other experiments.

Supplementary Information is linked to the online version of the paper at www.nature.com/nature.

The authors declare no competing financial interests.

The coordinates and structure factors have been deposited in the Protein Data Bank with accession numbers of 4JSN for the native structure, 4JSP for the ATP $\gamma \mathrm{S}-\mathrm{Mg}$ complex, 4JSV for the ADP-Mg-F complex, 4JSX for the Torin 2 complex, 4JT5 for the PP242 complex, and 4JT6 for the PI-103 complex. 
complexes also contain the requisite mLST8 subunit ${ }^{8,9}$, but they differ in a number of other subunits that interact with RAPTOR or RICTOR ${ }^{1}$.

mTORC1 regulates cell growth by promoting translation, ribosome biogenesis and autophagy ${ }^{1,4,5}$. Its activation requires nutrients and amino acids, which result in the RAPTOR-mediated recruitment of mTORC1 to lysosomes and late endosomes ${ }^{10,11}$, and colocalization with its activator, the small GTPase RHEB ${ }^{12,13}$. Proposed mechanisms of RHEB action include binding to and activating the mTOR kinase domain ${ }^{14}$, and the displacement of the mTORC1 inhibitor PRAS40 from RAPTOR ${ }^{15,16}$. RHEB in turn is negatively-regulated by the GTPase-activating domain of the TSC2 tumor suppressor, which relays signals from multiple growth factor and stress pathways ${ }^{17}$. mTORC1 substrates include the eIF4E-binding protein 1 (4EBP1) and ribosomal S6 kinases (S6K), which control cap-dependent translation initiation and elongation, respectively ${ }^{18}$. Phosphorylation of 4EBP1 and S6K1 is dependent on their Tor Signaling Sequence (TOS) motif ${ }^{19,20}$ that binds to RAPTOR, and which is also present in the negative regulator PRAS4021,22.

mTORC2 responds primarily to growth factors, promoting cell-cycle entry, cell survival, actin cytoskeleton polarization, and anabolic output ${ }^{6,7,23}$. Its substrates include the Ser/Thr protein kinases AKT, SGK and PKC, which share the hydrophobic motif (HM) phosphorylation site with $\mathrm{S} 6 \mathrm{~K} 1^{1,2}$.

Rapamycin, which forms a ternary complex with the FK506-binding protein12 (FKBP12) and the FRB (FKBP12-Rapamycin-Binding) domain of mTOR, is thought to be an allosteric inhibitor $^{24,25}$. Rapamycin-FKBP12 inhibits mTORC1 to a variable extent that is substrate and phosphorylation-site dependent ${ }^{25}$, and it does not bind to mTORC2 $2^{23}$. To overcome these limitations, ATP-competitive inhibitors that potently and uniformly inhibit both mTORC1 and mTORC2 are being developed as anti-cancer agents ${ }^{26}$.

The six mammalian PIKKs regulate diverse cellular processes ${ }^{27}$. They share three regions of homology consisting of a $\sim 600$ residue FAT domain (FRAP, ATM, TRRAP), a $\sim 300$ residue PI3K-related protein kinase catalytic domain, and a $\sim 35$ residue FATC domain at the Cterminus ${ }^{28}$. In mTOR, the $\sim 100$ residue FRB domain is thought to occur in-between the FAT and catalytic domains, and the region $\mathrm{N}$-terminal to the FAT domain is required for binding to RAPTOR and RICTOR ${ }^{1}$.

Here we present the $3.2 \AA$ crystal structure of a $\sim 1500$ amino acid mTOR-mLST8 complex containing the FAT, FRB, kinase and FATC domains, as well as the structures of this complex bound to an ATP transition state analog and to ATP-competitive inhibitors. We discuss their implications for understanding mTOR function, regulation and inhibition by rapamycin and ATP-competitive compounds.

\section{Overall structure of $\mathrm{mTOR}^{\Delta \mathrm{N}}-\mathrm{mLST8}$}

Crystals were grown using an N-terminally truncated human mTOR (residues 1376 to 2549; thereafter $\mathrm{mTOR}^{\Delta \mathrm{N}}$ ) bound to full-length human mLST8 (Supplementary Table 1). The complex was produced in an HEK293-F cell line that was stably-transfected sequentially by FLAG-tagged mLST8 and FLAG-tagged $\mathrm{mTOR}^{\Delta \mathrm{N}}$ vectors. The kinase activity of 
$\mathrm{mTOR}^{\Delta \mathrm{N}}$-mLST8 is overall comparable to that of mTORC1 (Supplementary Fig. 1). mTORC1 is more active towards low-micromolar concentrations of S6K1 ${ }^{\mathrm{ki}}$ (kinase-inactive mutant) and 4EBP1, consistent with RAPTOR recruiting these substrates through their TOS motifs, whereas $\mathrm{mTOR}^{\Delta \mathrm{N}_{-}}$-mLST8 is more active at higher substrate concentrations.

The $\mathrm{mTOR}^{\Delta \mathrm{N}}$-mLST8 structure has a compact shape (Fig. 1). The FAT domain, which consists of $a-a$ helical repeats, forms a " $C$ " shaped a solenoid that wraps halfway around the kinase domain and clamps onto it. mLST8 and the FRB domain protrude from the kinase domain, on opposite sides of the catalytic cleft. The FATC is integral to the kinase domain structure.

The 550-residue mTOR kinase domain (KD) adopts the two-lobe structure that is characteristic of both the PI3K and canonical protein kinase families ${ }^{29}$. It consists of an Nterminal lobe ( $\mathrm{N}$ lobe), a larger C-terminal lobe (C lobe), and a cleft in-between the two that binds to ATP. The mTOR KD contains the core PI3K KD fold, but with substantial differences. While PI3K KD structures superimpose essentially across their entire $\sim 350$ residues, only $\sim 250$ residues of the mTOR KD superimpose on PI3Ks (Supplementary Table 2). In addition, the mTOR KD contains $\sim 200$ additional residues of insertions that decorate the common fold. The largest of these is the FRB domain (residues 2021 to 2118) that is inserted within the kinase $\mathrm{N}$ lobe (Fig. 1). There is also a $~ 40$-residue insertion in the $\mathrm{C}$ lobe that forms the binding site for mLST8 (residues 2258 to 2296; thereafter LBE).

\section{Kinase domain structure}

The mTOR KD structure starts prior to the FRB domain, with the long ka1 helix that is present in PI3Ks as well (Fig. 2a and Supplementary Fig. 2a). The ka1 helix is integral to the structure of the $\mathrm{N}$ lobe, as it packs in the concave surface of the $\mathrm{N}$ lobe $\beta$ sheet in both mTOR and PI3Ks ${ }^{29}$. The FRB insertion occurs immediately after ka1. It is followed by a $\beta$ strand and two short helices that pack with the base of the FRB, and which replace the ka2 helix of the PI3K KD fold. Thereafter, the mTOR and PI3K N lobes share a similar structure.

The mTOR structure indicates that the ka1 helix is present in all PIKKs (Supplementary Fig. 3a). The SMG1, DNAPKcs and TRRAP PIKKs also appear to have an FRB-like domain, as they contain insertions of 128,95 and 128 residues, respectively, that are predicted to be alpha-helical. This is supported by the $6.6 \AA \mathrm{x}$-ray diffraction data of DNAPKcs reported recently ${ }^{30}$. Although the deposited DNAPKcs model, which contains a PI3K-derived KD, lacks the FRB insertion, a reinterpretation of the data using the mTOR structure reveals electron density indicative of an FRB-like four-helix bundle (Supplementary Fig. 3b). ATM and ATR appear to lack a comparable insertion.

In the $\mathrm{C}$ lobe, the vicinity of the catalytic cleft contains four structural insertions compared to PI3Ks (LBE, kaAL, ka9b and FATC; Fig. 2a). These form a spine of interactions centered on the activation loop, which is a $\sim 30$ residue segment with a central role in the function and regulation of canonical protein kinases. It forms part of the polypeptide binding site, provides an active site residue, and also undergoes a conformational change, typically disorder-to-order transition, upon kinase activation ${ }^{31}$. The activation loop is thought to have 
an analogous role in PI3Ks, among which only in the class 3 PIK3C3/Vps34 structures it is ordered $^{32}$. In the mTOR structure the entire activation loop is well ordered (Supplementary Fig. 2b), and its middle portion contains the kaAL helix insertion. One side of the activation loop packs with the ka9b insertion, and the other side with the FATC (Fig. 2a). The FATC is not unique to PIKKs in its entirety, as its N-terminal half forms a helix (ka11) that is also present in PI3K structures ${ }^{29,32}$. Its C-terminal half, which is absent from PI3Ks, forms three short helices that pack with the activation loop on one side and the LBE on the other (Fig. 2a).

The interactions the FATC makes with the activation loop suggest that it may have a role in stabilizing the activation loop structure, and the LBE, through its interactions with the FATC, may contribute to this indirectly (Supplementary Fig. 4a). The FATC and activation loop sequences are highly conserved among PIKKs. While the LBE is not conserved, all PIKK family members contain an LBE-like insertion that may similarly pack with FATC (Supplementary Fig. 3a).

The ka9b insertion (residues 2425 to 2436) plugs one end of the catalytic cleft (Fig. 2a). It partially overlaps with a segment, termed negative regulatory domain (residues 2430 to 2450), whose deletion activates mTOR in vitro ${ }^{33}$ and in vivo ${ }^{34,35}$. After ka9b, there is a 55 residue unstructured segment (2437 to 2491) that is not conserved and has variable length in mTOR orthologs (Supplementary Fig. 2a).

\section{Active site conformation}

To assess the activation state of the KD structure and investigate the mechanism of phosphotransfer, we co-crystallized mTOR ${ }^{\Delta \mathrm{N}}$-mLST8 with ADP, $\mathrm{Mg}^{2+}$ and $\mathrm{MgF}_{3}{ }^{-}$, a mimic of the $\gamma$-phosphate group of ATP in the transition state (TS) ${ }^{36}$ (Supplementary Table 1). The $3.5 \AA F o-F c$ map of these crystals shows strong electron density extending from the $\beta$-phosphate group of ADP, consistent with a bound $\mathrm{MgF}_{3}{ }^{-}$group (Fig. 2b). The presence of two additional $\mathrm{Mg}^{2+}$ ions is supported by the anomalous dispersion maps of apo-crystals soaked in AMPPNP-Mn ${ }^{2+}$ (Supplementary Fig. 4b).

A superposition with the protein kinase CDK2 bound to the same ATP TS mimic ${ }^{36}$ reveals that the arrangement of key active site residues and $\mathrm{MgF}_{3}{ }^{-}$is remarkably conserved in mTOR (Fig. 2c and Supplementary Fig. 4c). These include the C lobe Asn2343 and Asp2357, which in both mTOR and protein kinases serve as metal ligands, and Asp2338, which in protein kinases plays a key catalytic role in orienting and activating the substrate hydroxyl group for nucleophilic attack ${ }^{36,37}$. The mTOR-CDK2 superposition also reveals a coincidence of the mTOR His 2340 and CDK2 Lys129 side chains (Fig. 2c). In protein kinases, a basic residue at this position interacts with both the substrate hydroxyl group and the $\gamma$-phosphate TS mimic, and is postulated to stabilize the buildup of charge at the transition state ${ }^{36,37}$. Consistent with a similar role, we find that the kinase activity of the $\mathrm{H} 2340 \mathrm{~A} \mathrm{mTOR}^{\Delta \mathrm{N}_{-}}$-mLST8 mutant is barely detectable, and comparable to the D2338A mutant previously shown to be inactive ${ }^{38}$ (Supplementary Fig. 4d).

These findings strongly suggest that the crystallized mTOR ${ }^{\Delta \mathrm{N}}$-mLST8 complex is intrinsically active in the absence of any additional regulatory subunits. They also indicate 
that PIKKs, and most likely PI3Ks, employ the same catalytic mechanism as canonical protein kinases, in contrast to a recently proposed PI3K mechanism ${ }^{32}$ (see legend of Supplementary Fig. 4c).

\section{Substrate-binding site}

The $\mathrm{MgF}_{3}{ }^{-} \gamma$-phosphate mimic points to an extended $\mathrm{C}$ lobe groove that is highly conserved and is the likely site of substrate binding. Figure $2 \mathrm{~d}$ illustrates this with a CDK2-bound substrate peptide docked by superposing the mTOR and CDK2 TS $^{36}$ complexes. The groove consists of the activation loop, as in protein kinases, but also of portions of the FATC and LBE.

In the (+) direction (C-terminal to phosphorylation site), the groove extends only to the +1 position, abruptly terminating with the ka9b helix that plugs this end of the cleft (Fig. 2d). Thereafter, the peptide can exit along the C lobe towards mLST8, or along the $\mathrm{N}$ lobe towards the FRB. The +1 portion of the groove has a pronounced pocket lined with three aromatic residues, consistent with the two classes of mTOR substrates, exemplified by 4EBP1 and S6K1, having a proline and tyrosine residue, respectively, at +1 (Supplementary Figs $5 \mathrm{a}$ and $5 \mathrm{~b}$ ). The groove extends substantially more in the (-) direction, and it may account for the low-level sequence preference at -4 and -5 suggested by a positional scanning peptide array ${ }^{39}$.

\section{Restricted access to the active site}

The four-helix FRB substantially extends the $\mathrm{N}$ lobe side of the catalytic cleft, while the LBE and mLST8 extend the C lobe side. This gives the cleft a deep, "V"-shaped crosssection, restricting access to the substrate-binding site at the bottom of the cleft (Fig. 3a). Substrate access is further hindered by one end of the cleft being plugged by ka9b and the following 55-residue unstructured segment. Portions of ka9b and the unstructured segment form the negative regulatory region ${ }^{33-35}$, suggesting that restricted access to the active site negatively regulates mTOR. Ka9b is likely the key element because deleting the bulk of the unstructured segment (residues 2443-2486) does not activate mTOR (Supplementary Fig. 6).

The end of the cleft opposite ka9b is unencumbered in the mTOR ${ }^{\Delta \mathrm{N}}$-mLST8 structure. However, it is near the FAT N-terminus, and the region deleted from the crystallized $\mathrm{mTOR}^{\Delta \mathrm{N}}$ may restrict cleft access directly and/or through its associated RAPTOR or RICTOR proteins. In support, we find that towards TOS-mutant 4EBP1 and S6K1 ${ }^{\mathrm{ki}}$ substrates, $\mathrm{mTOR}^{\triangle \mathrm{N}}$-mLST8 is more active than mTOR-mLST8, which in turn is more active than mTOR-mLST8-RAPTOR (Supplementary Fig. 6). A restricted active site would reduce the probability of substrates hitting the active site by random diffusion, and could thus be important in allowing for the regulation of phosphorylation by substrate-recruitment.

\section{Inhibition by rapamycin-FKBP12}

In a model constructed by superposing the FRB domains of the FRB-rapamycin-FKBP12 24 and $\mathrm{mTOR}^{\Delta \mathrm{N}}$-mLST8 structures, FKBP12 extends from the FRB towards mLST8 on the C lobe, nearly capping the catalytic cleft (Fig. 3b). At their closest approach, FKBP12 and 
mLST8 are only $8 \AA$ apart, with the catalytic center recessed by $37 \AA$. The model suggests that rapamycin-FKBP12 inhibits in part by drastically reducing the accessibility of the already constricted catalytic cleft. This is consistent with the extent of mTORC1 inhibition being substrate and phosphorylation site dependent ${ }^{25}$ (additional discussion in Supplementary Fig. 6 legend).

\section{FRB recruits S6K1 to active site}

The rapamycin-binding site maps to the FRB surface closest to the active site, in the midst of a hotspot of highly conserved residues (Fig. 4a). Four of these residues are invariant in 22 mTOR orthologs from yeast to man, and another four in 21 orthologs, in stark contrast to the FRB surface opposite from the active site (Fig. 4a and Supplementary Fig. 7). This raises the possibility that the rapamycin-binding site interacts with substrates to facilitate their entry to the recessed active site.

This model is supported by the observation that mutation of Ser2035, a rapamycin contact $^{38,40}$ at the centre of the conservation hotspot, reduces phosphorylation of S6K1 and $4 \mathrm{EBP} 1^{33}$. It can also explain inhibition of mTORC 1 and $\mathrm{mTORC} 2$ by rapamycin in the absence of FKBP12, albeit at $\sim 100$-fold higher concentrations reflecting the weaker rapamycin-FRB binary interaction ${ }^{41}$. However, these observations can also be explained by the rapamycin-binding site interacting with other parts of mTOR, or with associated proteins, especially since rapamycin was thought to be an allosteric inhibitor ${ }^{25}$. To rule this out, we assayed $\mathrm{mTOR}^{\Delta \mathrm{N}}$-mLST8, and found that rapamycin but not the closely-related FK506 macrolide inhibits $\mathrm{S} 6 \mathrm{~K} 1{ }^{\mathrm{ki}}$ overall phosphorylation, as measured by ${ }^{32} \mathrm{P}$ incorporation (Fig. 4b). Inhibition reaches $\sim 45 \%$ at the $20 \mu \mathrm{M}$ solubility limit of rapamycin, close to the free rapamycin-FRB $\mathrm{K}_{\mathrm{d}}$. Phosphorylation of the key Thr389 site of the S6K1 HM motif, measured by a phosphoT389-specific antibody, is inhibited by $\sim 80 \%$ (Fig. 4b).

In a complementary approach, we tested whether the isolated FRB domain inhibits phosphorylation in trans. Figure $4 \mathrm{c}$ shows that wild type but not the S2035I FRB mutant ${ }^{38,40}$ inhibits overall S6K1 $1^{\mathrm{ki}}$ phosphorylation by $\sim 50 \%$ and $\mathrm{Thr} 389$ phosphorylation by $\sim 75 \%$ at the highest concentration tested.

To map the region of S6K1 involved in FRB interactions, we reasoned that its deletion should reduce Thr389 phosphorylation and also render any residual phosphorylation insensitive to rapamycin. By testing successively truncated polypeptides from the S6K1 tail, we found that both criteria are met by residues 393 to 398 (Fig. 4d). In addition, residues 399 to 402 and 403 to 410 likely contribute to FRB interactions, as their truncation progressively reduces phosphorylation (Supplementary Fig. 8). N-terminal truncations up to residue 381 have no significant effect.

Together, these data indicate that the FRB provides a secondary substrate-recruitment site near the entrance of the catalytic cleft. We presume that while the TOS and possibly other motifs are the primary means of substrate recruitment, the secondary site may facilitate substrate entry into the otherwise restricted active site, and it may also provide an additional level of specificity, at least for a subset of substrates. 
A bi-partite substrate-recruitment mechanism is analogous to what has been proposed for the calcineurin phosphatase, the target of FK506-FKBP12. Most calcineurin substrates require both a primary and a secondary recruitment motif for efficient dephosphorylation, and FK506-FKBP12 inhibits by binding to the secondary recruitment motif site ${ }^{42}$.

\section{FAT domain}

The FAT domain contains 28 a helices arranged as $\alpha-a$ helical repeats. Helices $a 1$ to a22 belong to the TPR repeat family and form three discontinuous domains (TRD1, TRD2 and TRD3). Helices a 23 to a 28 belong to the HEAT family and form a single domain (HRD). The four domains pack sequentially to form a C-shaped a solenoid that clamps onto the KD (Supplementary Figs 9a and 9b). TRD1 interacts with the C lobe on one side of the KD, and after TRD2-TRD3 traverse to the other side, the HRD interacts with both the $\mathrm{N}$ and $\mathrm{C}$ lobes of the KD (Supplementary Figs 9c and 9d).

Both the TRD1-KD and HRD-KD contacts involve conserved residues, consistent with the two interfaces being important for the structure and function of mTOR. For example, a set of buried hydrogen bond contacts at the TRD1-KD interface (Glu1401-Arg2317) and at the HRD-KD interface (Arg1905-Glu2419 and Gln1941- Gln2200) involves residues invariant in 22 mTOR orthologs (Supplementary Figs 9c and 9d).

The TRD1 and HRD segments correspond to the FAT segments best conserved among PIKK family members ${ }^{28}$ as well, suggesting that the FAT domain clamping onto the KD is a common feature of this family. In addition, PI3Ks contain a HEAT-repeat domain analogous to the HRD in its structure and interactions with the KD, although they lack the rest of the FAT domain (Supplementary Fig. 9e).

\section{mLST8}

mLST8 consists of seven WD40 repeats. At the narrow end of the mLST8 $\beta$ propeller structure, a surface that extends across six WD40 repeats binds to both helices and intervening loop of the LBE helix-loop-helix structure. The interface involves mostly polar LBE residues and polar/aromatic mLST8 residues, and it is dominated by hydrogen bonds (Supplementary Fig. 10).

mLST8 is thought to be a requisite activating subunit of mTOR complexes ${ }^{8,9}$. The structure suggests the extended interaction surface of mLST8 may directly stabilize the LBE structure, and indirectly influence the organization of the active site through the LBEFATC-catalytic loop spine of interactions (Supplementary Fig. 4a). In support, we note that lack of mLST8 results in the association of mTOR with heat shock proteins ${ }^{9}$, and we find that the solubility of overexpressed mTOR is highly dependent on mLST8 co-expression (not shown). In addition, the growth-suppression phenotype of a temperature-sensitive yeast Lst8 allele can be rescued by mutations ${ }^{43}$ either at the LBE hydrophobic core (A2290V) or at the following ka5 helix (L2302Q), and the structure is consistent with these mutations stabilizing the local LBE structure. 


\section{Control of mTOR kinase activity}

The model that active site restriction is a negative regulatory mechanism is supported by hyper-activating mutations ${ }^{43-45}$, most of which map to structural elements involved in limiting active site access. A large number of mutations cluster at the end of the catalytic cleft that is plugged by ka9b (Fig. 5 and Supplementary Fig. 11). They map to the ka3, ka9, $\mathrm{ka} 9 \mathrm{~b}$ and ka10 helices that pack extensively with each other, and to the FAT HRD portion that packs with ka3 and ka9. These mutations are likely to loosen the ka9b-centered structural framework that restricts access from this end of the cleft, presumably without destabilizing the entire active site structure. The role of the FAT clamp is highlighted by the widely studied E2419K activating mutation, which eliminates the Glu2419-Arg 1905 salt bridge at the ka9-HRD interface (Supplementary Figs 9c and 11).

Two other hyper-activating mutations map to a portion of the ka1 helix that is sandwiched between the FRB base and the $\mathrm{N}$ lobe $\beta$ sheet $^{43,44}$. These mutations (I2017V and A2020V) are unlikely to unfold the FRB, because an FRB hydrophobic core mutation (W2027F) abolishes kinase activity ${ }^{40}$. The structure suggests that these mutations may loosen the rigid coupling of the FRB the catalytic cleft, increasing access to the active site.

A third cluster of mutations ${ }^{43}$ maps to the $\mathrm{N}$-terminal portion of the FAT domain, to structural residues of TRD1 and of the TRD1-proximal portion of TRD2. These mutations would likely destabilize TRD1-KD interactions through loss of structural integrity. They may also destabilize the HRD-KD interactions, since the structural coupling of TRD1 and HRD would thermodynamically couple their KD contacts. In addition, the disposition of the $\mathrm{N}$-terminal mTOR segment and its associated RAPTOR could change or become more flexible relative to the KD.

\section{Inhibitors of the ATP-binding site}

To explore the determinants of inhibitor potency and specificity, we determined the structures of mTOR ${ }^{\Delta N_{-}}$mLST8 bound to Torin2 and PP242, which are highly specific for mTOR, and to PI-103, which has dual specificity for mTOR and Class I PI3Ks (Supplementary Table 3).

The overall orientation of Torin 2 in the ATP site is as predicted ${ }^{46}$ (Fig. 6a and Supplementary Fig. 12a). The tricyclic benzonapththyridine ring binds to the adenine site and makes a hydrogen bond to the N-C lobe "hinge", analogous to one of two backbone hydrogen bonds made by ATP and diverse PI3/protein kinase inhibitors. The amino-pyridine group reaches into the "inner hydrophobic pocket", an area in the back of the cleft that many kinase inhibitors contact. However, it does not make the three predicted hydrogen bonds (to Asp2195, Asp2357 and Tyr2225) ${ }^{46}$. Rather, the structure reveals that a key interaction is the extensive stacking of the tricyclic benzonapththyridine ring with the indole group of Trp2239 from the "hinge" (Fig. 6a). In the ATP $\gamma$ S complex, Trp2239 stacks partially with a three-atom portion of the adenine. In the Torin 2 complex, by contrast, Trp2239 stacks with a ten-atom portion of the tricyclic Troin 2 ring. This likely makes a substantial contribution to the sub-nanomolar potency ${ }^{46}$ of Torin2. In addition, because Trp2239 is not present in canonical protein kinases or in PI3Ks, it would also contribute to Torin2's 800-fold 
specificity for mTOR over PI3Ks ${ }^{46}$. The structure also reveals that the Torin2 trifluoromethyl group packs into an N lobe pocket (Ile2163, Pro2169 and Leu2185; Fig. 6a). These contacts are less extensive than those made to Trp2239, and only Leu2185 is variable across the PIKK and PI3K families. They may contribute to Torin2-mTOR specificity, however, as this portion of the $\mathrm{N}$ lobe $\beta$ sheet exhibits considerable variation in its relative orientation in PI3K structures.

PP242 consists of the adenine-mimetic pyrazolopyrimidine scaffold common to PI3/tyrosine kinase inhibitors, with a hydroxyindole substituent at a position that often points to the inner hydrophobic pocket ${ }^{47}$. In mTOR, this pocket is lined with residues conserved across the PI3K and PIKK families, and the selectivity of PP242 for mTOR was unexpected ${ }^{47}$. The structure reveals that while these two PP242 groups are generally positioned as predicted ${ }^{47}$, mTOR undergoes a conformational change that expands and deepens the inner hydrophobic pocket, with the PP242 hydroxyindole group reaching deep into the new space (Fig. 6b and Supplementary Fig. 12b). The conformational change involves the Tyr2225 side chain at the back of the pocket swinging $\left(\Delta_{\chi_{1}}=108^{\circ}\right)$ out of the way of the hydroxyindole group (Fig. 6c and Supplementary Fig. 12c). Because Tyr2225 is part of the local hydrophobic core between the $\mathrm{N}$ and $\mathrm{C}$ lobes, this necessitates additional concerted changes. The Leu2354 side chain rotates $\left(\Delta \chi_{1}=166^{\circ}\right)$ away from the incoming Tyr2225, the Gln2223 side chain moves in to plug a resulting gap, and a 3-residue main chain stretch (residues 2223 to 2225) shifts by $\sim 1$ A. In PI3Ks, Leu2354 is replaced by a conserved phenylalanine that cannot undergo a similar conformational change, at least without an even more extensive repacking of the local hydrophobic core. Leu2354 is thus likely to be a major, though indirect, determinant of the selectivity of PP242 for mTOR over PI3Ks and other kinases.

The multi-targeted PI- $103^{48}$ does not take advantage of any of the aforementioned mTORspecific features. The morpholine ring binds to the adenine pocket and makes two hydrogen bonds to the "hinge", while the $m$-phenol group binds to the inner pocket and makes two hydrogen bonds to the Tyr2225 and Asp2195 side chains at the back of the cleft (Fig. 6d and Supplementary Fig. 12d). These hydrogen bonds are likely to be important for the high affinity of PI-103 for mTOR and, presumably, for Class I PI3Ks where the relevant protein groups are similarly positioned. In support, we note that the $\sim 500$-fold weaker affinity ${ }^{48}$ Class III PI3K-PI-103 complex lacks both $m$-phenol hydrogen bonds and one of the "hinge" hydrogen bonds ${ }^{32}$.

\section{Conclusions}

The $\mathrm{mTOR}^{\Delta \mathrm{N}}$ structure reveals an intrinsically active but otherwise highly restricted catalytic centre, and together with activating mutations points to substrate recruitment as a major mechanism controlling the kinase activity. Our biochemical data on S6K1 indicates that in addition to a primary recruitment motif, a secondary recruitment motif proximal to the phosphorylation site is needed for efficient phosphorylation. The FRB acts as a gatekeeper by restricting access to the active site while also granting privileged substrates access through its binding site for the secondary motif. Co-crystal structures of ATPcompetitive inhibitors reveal multiple determinants of mTOR specificity, including a conformational change deep inside the catalytic cleft. 


\section{Methods}

\section{Protein expression and purification}

The boundary of the crystallized human $\mathrm{mTOR}^{\Delta \mathrm{N}}$ (residues 1376-2549) was identified by screening successive $\mathrm{N}$-terminal truncations of mTOR, produced by transient transfection in HEK293-F cells, for expression levels, solubility and size exclusion chromatography profile (not shown). For large-scale production of $\mathrm{mTOR}^{\Delta \mathrm{N}_{-}}$mLST8, we generated an HEK 293-F cell line that was stably-transfected sequentially by modified pcDNA3.1 vectors encoding FLAG-tagged mLST8 and FLAG-tagged $\mathrm{mTOR}^{\Delta \mathrm{N}}$. In the absence of mLST8 coexpression, the level of soluble $\mathrm{mTOR}^{\Delta \mathrm{N}}$ was substantially lower, and it co-purified with endogenous mLST8 (not shown). The mTOR ${ }^{\Delta \mathrm{N} \Delta-2486}$-mLST8, full-length human mTORmLST8 and human mTORC1 (mTOR-mLST8-RAPTOR) complexes were similarly produced in HEK 293-F cell lines that were stably transfected with the FLAG-tagged versions of the proteins. Cells were grown as monolayers in 15 centimeter plates. They were lysed in 50mM Tris-Cl, pH 8.0, $100 \mathrm{mM} \mathrm{KCl}, 400 \mathrm{mM} \mathrm{NaCl}, 1 \mathrm{mM}$ EDTA, 1 mM EGTA, $10 \%$ (v/v) glycerol, $2 \mathrm{mM}$ dithiothreitol (DTT) and protease inhibitors using French Press. After centrifugation, the FLAG-tagged proteins were affinity purified using anti-FLAG M2 agarose beads (Sigma). Following cleavage of the FLAG-tags by tobacco etch virus (TEV) protease, they were purified by ion exchange (MonoQ) and gel-filtration chromatography. The purified complex was concentrated to $5 \mathrm{mg} \mathrm{ml}^{-1}$ by ultrafiltration in $20 \mathrm{mM}$ Tris-Cl, $500 \mathrm{mM} \mathrm{NaCl}, 10 \mathrm{mM}$ DTT, pH 8.0 and was used for crystallization immediately. For kinase assays, $10 \%(\mathrm{v} / \mathrm{v})$ glycerol was added to protein aliquots for storage at $-80{ }^{\circ} \mathrm{C}$.

Human S6K1aII ${ }^{\mathrm{ki}}$ protein, which contains the kinase-inactivating K100R mutation, was produced by infecting High Five insect cells with a pFastBac1 baculovirus expressing the FLAG-tagged protein. It was purified by affinity chromatography with anti-FLAG M2 agarose beads. The protein was concentrated to $8 \mathrm{mg} \mathrm{ml}^{-1}$ in $50 \mathrm{mM}$ Tris-Cl, $400 \mathrm{mM} \mathrm{NaCl}$, $1 \mathrm{mM}$ EDTA, 1mM EGTA, 5\% (v/v) glycerol, $10 \mathrm{mM}$ DTT, $1 \mathrm{mM}$ Pefabloc, pH 8.0, and it was stored at $-80^{\circ} \mathrm{C}$.

Human 4EBP1 was overexpressed in the E. Coli strain BL21(DE3) from a modified pGEX4T3 vector, and was purified by glutathione affinity chromatography, cleavage of the GST tag with TEV protease, fractionation on MonoQ column, removal of free GST by glutathione affinity, and finally gel filtration (Superdex75) chromatography. The peak fractions were concentrated to $20 \mathrm{mg} \mathrm{ml}^{-1}$ in $20 \mathrm{mM}$ Tris-Cl, $200 \mathrm{mM} \mathrm{NaCl}, 5 \%$ (v/v) glycerol, 10 mM DTT, pH 8.0.

\section{Crystallization and data collection}

Crystals were grown by the hanging-drop vapour diffusion method at $4{ }^{\circ} \mathrm{C}$. Apo-crystals of the $\mathrm{mTOR}^{\Delta \mathrm{N}}$-mLST8 complex were grown from $100 \mathrm{mM}$ Tris-Cl, 6-8\% (w/v) polyethylene glycol (PEG) 8000, $500 \mathrm{mM} \mathrm{NaCl}, 10 \%$ (v/v) glycerol, $10 \mathrm{mM} \mathrm{DTT}$, pH 8.5. Crystals of the $\mathrm{mTOR}^{\Delta \mathrm{N}_{-}}$-mLST8 bound to ADP-MgF $3-\mathrm{Mg}_{2}$ were grown similarly, except the well-buffer

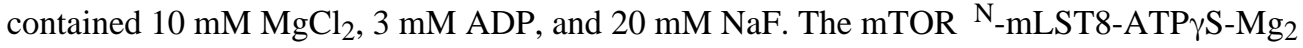
complex was prepared by soaking apo-crystals for one hour in a stabilization buffer of 50 $\mathrm{mM}$ Tris-Cl, pH 8.5, $10 \mathrm{mM}$ Tris-Cl, 8.0, 10\% PEG8000, 0.1 M NaCl, 6\% glycerol, 
supplemented with $5 \mathrm{mM} \mathrm{MgCl}_{2}$ and $1 \mathrm{mM}$ ATP $\gamma \mathrm{S}$. The $\mathrm{mTOR}^{\Delta \mathrm{N}}-\mathrm{mLST}$-AMPPNP-Mn complex was prepared by soaking apo-crystals similarly, except the stabilization buffer had a pH of 7.5 and it was supplemented with $1 \mathrm{mM}$ AMPPNP and $2 \mathrm{mM} \mathrm{MnCl}_{2}$, and the data was collected at the Manganese absorption edge. Crystals of $\mathrm{mTOR}^{\Delta \mathrm{N}}-\mathrm{mLST}$-Torin2 and $\mathrm{mTOR}^{\Delta \mathrm{N}}$-mLST8-PI-103 were prepared by mixing $1 \mathrm{mM}$ of the inhibitors with the protein. Co-crystals appeared from the same condition as the apo-crystals. Crystals of mTOR ${ }^{\Delta \mathrm{N}_{-}}$ mLST8-PP242 were prepared by soaking apo-crystals for 2.5 hours in the stabilization buffer supplemented with $0.2 \mathrm{mM}$ PP242. Apo-crystals were harvested in stabilization buffer, transferred to $50 \mathrm{mM}$ Tris-Cl, $\mathrm{pH} 8.5,10 \mathrm{mM}$ Tris-Cl, $\mathrm{pH}$ 8.0, $0.1 \mathrm{M} \mathrm{NaCl}, 14 \%$ (w/v) PEG8000, 22\% (v/v) glycerol, and were flash-frozen in liquid nitrogen. Crystals with $\mathrm{ADP}-\mathrm{MgF}_{3}-\mathrm{Mg}_{2}, \mathrm{ATP} \gamma \mathrm{S}-\mathrm{Mg}_{2}$, Torin2, PI103 and PP242 were flash-frozen similarly, except for the presence of the corresponding cofactors or inhibitors $(0.1 \mathrm{mM})$ in the buffers. Diffraction data were collected at $-170{ }^{\circ} \mathrm{C}$ at the ID24C and ID24E beamlines of the Advanced Photon Source, and they were processed with the HKL suite ${ }^{49}$.

\section{Structure determination and refinement}

Initial phases were obtained from isomorphous and anomalous differences of two heavy atom derivatives, prepared by soaking crystals in stabilization buffer lacking DTT and supplemented with $1 \mathrm{mM}$ uranyl acetate (1 hour), or with $0.4 \mathrm{mM}$ potassium-gold cyanide (75 min.) at at $4{ }^{\circ} \mathrm{C}$. Initial phases, calculated with the program SHARP ${ }^{50}$ had a mean figure of merit of 0.35 (35.0 to $4.5 \AA$ ). The uranyl derivative had a dispersive phasing power $(P p)$ of 0.89 and anomalous $P p$ of 0.60 , with a dispersive $R_{\text {cullis }}$ of 0.79 and anomalous $R_{\text {cullis }}$ of 0.95 . The gold derivative had a dispersive $P p$ of 0.80 and anomalous $P p$ of 0.29 , with a dispersive $R_{\text {cullis }}$ of 0.66 and anomalous $R_{\text {cullis }}$ of 0.98 . The phases were improved using solvent flattening and two-fold ncs averaging with multiple masks with the program $\mathrm{DM}^{51}$. The model was built using $\mathrm{O}^{52}$ and refined first with REFMAC $5^{51}$ and then with PHENIX $^{53}$, using tight ncs restraints on atom positions. The final model contains residues 1385 to 2549 of human mTOR, and 8 to 324 of human $\mathrm{mLST}$. $\mathrm{mTOR}^{\Delta \mathrm{N}}$ residues 1376-1384 at the N-terminus, residues 1815-1866 in the FAT domain, and residues 2437-2491 between ka9b and ka10 in the KD are disordered. mLST8 residues 1-7 and 325-326 from the N- and C-termini are disordered. The Ramachandran plot, calculated by PROCHECK, has $88.5,11.0$ and $0.5 \%$ of the residues in the most favored, additionally allowed, and generously allowed regions, respectively. There are no residues in disallowed regions. The $R_{\text {free }}$ test set of the Native data contains 1699 reflections.

\section{In vitro kinase assays}

All kinase assays were performed in a buffer of $25 \mathrm{mM}$ HEPES, pH 7.4, $100 \mathrm{mM} \mathrm{NaCl}, 10$ $\mathrm{mM} \mathrm{MgCl} 2,2 \mathrm{mM}$ DTT, $3 \%$ (v/v) glycerol, for 30 minutes at $30^{\circ} \mathrm{C}$, in a final volume of 15 $\mu l$. For a typical reaction, the indicated amount of enzymes, substrates, and competitors were incubated together for 10 minutes on ice in the kinase buffer in a final volume of $13.5 \mu$. Reactions were started by the addition of $1.5 \mu \mathrm{l}$ of cold ATP $(0.5 \mathrm{mM}$ final concentration) including $2 \mu \mathrm{Ci}$ of $\left[\gamma_{-}{ }^{32} \mathrm{P}\right]$ ATP $(6000 \mathrm{Ci} / \mathrm{mmol}$, Perkin-Elmer). In assays with rapamycin, all reactions in a titration contained a constant level of $3.3 \%$ DMSO. Reactions were stopped by the addition of $15 \mu \mathrm{l}$ of $2 \mathrm{X}$ NuPAGE LDS sample buffer and boiling for 3 minutes, and were resolved in a 4-12 \% NuPAGE Bis-Tris gel. Where applicable, each 
reaction was split into two for analysis by phosphorimaging and immunoblotting. All antibodies were obtained from Cell Signaling, except for the anti-FLAG antibody which was obtained from Sigma.

\section{Supplementary Material}

Refer to Web version on PubMed Central for supplementary material.

\section{Acknowledgments}

We thank D. King for mass spectroscopic analysis; H. Erdjument-Bromage for N-terminal sequencing; the staff of the Advanced Photon Source ID24 beamlines for help with data collection; Dorothea Robev for help with protein expression in HEK 293 cells. This work was supported by the Howard Hughes Medical Institute.

\section{References}

1. Zoncu R, Efeyan A, Sabatini DM. mTOR: from growth signal integration to cancer, diabetes and ageing. Nat Rev Mol Cell Biol. 2011; 12:21-35. [PubMed: 21157483]

2. Shaw RJ, Cantley LC. Ras, PI(3)K and mTOR signalling controls tumour cell growth. Nature. 2006; 441:424-430. [PubMed: 16724053]

3. Keith CT, Schreiber SL. PIK-related kinases: DNA repair, recombination, and cell cycle checkpoints. Science. 1995; 270:50-51. [PubMed: 7569949]

4. Hara K, et al. Raptor, a binding partner of target of rapamycin (TOR), mediates TOR action. Cell. 2002; 110:177-189. [PubMed: 12150926]

5. Kim DH, et al. mTOR interacts with raptor to form a nutrient-sensitive complex that signals to the cell growth machinery. Cell. 2002; 110:163-175. [PubMed: 12150925]

6. Loewith $\mathrm{R}$, et al. Two TOR complexes, only one of which is rapamycin sensitive, have distinct roles in cell growth control. Mol Cell. 2002; 10:457-468. [PubMed: 12408816]

7. Sarbassov DD, et al. Rictor, a novel binding partner of mTOR, defines a rapamycin-insensitive and raptor-independent pathway that regulates the cytoskeleton. Curr Biol. 2004; 14:1296-1302. [PubMed: 15268862]

8. Chen EJ, Kaiser CA. LST8 negatively regulates amino acid biosynthesis as a component of the TOR pathway. J Cell Biol. 2003; 161:333-347. [PubMed: 12719473]

9. Kim DH, et al. GbetaL, a positive regulator of the rapamycin-sensitive pathway required for the nutrient-sensitive interaction between raptor and mTOR. Mol Cell. 2003; 11:895-904. [PubMed: 12718876]

10. Sancak Y, et al. The Rag GTPases bind raptor and mediate amino acid signaling to mTORC1. Science. 2008; 320:1496-1501. [PubMed: 18497260]

11. Kim E, Goraksha-Hicks P, Li L, Neufeld TP, Guan KL. Regulation of TORC1 by Rag GTPases in nutrient response. Nat Cell Biol. 2008; 10:935-945. [PubMed: 18604198]

12. Saucedo LJ, et al. Rheb promotes cell growth as a component of the insulin/TOR signalling network. Nat Cell Biol. 2003; 5:566-571. [PubMed: 12766776]

13. Stocker H, et al. Rheb is an essential regulator of S6K in controlling cell growth in Drosophila. Nat Cell Biol. 2003; 5:559-565. [PubMed: 12766775]

14. Long X, Lin Y, Ortiz-Vega S, Yonezawa K, Avruch J. Rheb binds and regulates the mTOR kinase. Curr Biol. 2005; 15:702-713. [PubMed: 15854902]

15. Sato T, Nakashima A, Guo L, Tamanoi F. Specific activation of mTORC1 by Rheb G-protein in vitro involves enhanced recruitment of its substrate protein. J Biol Chem. 2009; 284:12783-12791. [PubMed: 19299511]

16. Sancak Y, et al. PRAS40 is an insulin-regulated inhibitor of the mTORC1 protein kinase. Mol Cell. 2007; 25:903-915. [PubMed: 17386266]

17. Zhang Y, et al. Rheb is a direct target of the tuberous sclerosis tumour suppressor proteins. Nat Cell Biol. 2003; 5:578-581. [PubMed: 12771962] 
18. Ma XM, Blenis J. Molecular mechanisms of mTOR-mediated translational control. Nat Rev Mol Cell Biol. 2009; 10:307-318. [PubMed: 19339977]

19. Schalm SS, Fingar DC, Sabatini DM, Blenis J. TOS motif-mediated raptor binding regulates 4EBP1 multisite phosphorylation and function. Curr Biol. 2003; 13:797-806. [PubMed: 12747827]

20. Nojima H, et al. The mammalian target of rapamycin (mTOR) partner, raptor, binds the mTOR substrates p70 S6 kinase and 4E-BP1 through their TOR signaling (TOS) motif. J Biol Chem. 2003; 278:15461-15464. [PubMed: 12604610]

21. Oshiro N, et al. The proline-rich Akt substrate of $40 \mathrm{kDa}$ (PRAS40) is a physiological substrate of mammalian target of rapamycin complex 1. J Biol Chem. 2007; 282:20329-20339. [PubMed: 17517883]

22. Fonseca BD, Smith EM, Lee VH, MacKintosh C, Proud CG. PRAS40 is a target for mammalian target of rapamycin complex 1 and is required for signaling downstream of this complex. J Biol Chem. 2007; 282:24514-24524. [PubMed: 17604271]

23. Jacinto E, et al. Mammalian TOR complex 2 controls the actin cytoskeleton and is rapamycin insensitive. Nat Cell Biol. 2004; 6:1122-1128. [PubMed: 15467718]

24. Choi J, Chen J, Schreiber SL, Clardy J. Structure of the FKBP12-rapamycin complex interacting with the binding domain of human FRAP. Science. 1996; 273:239-242. [PubMed: 8662507]

25. Choo AY, Blenis J. Not all substrates are treated equally: implications for mTOR, rapamycinresistance and cancer therapy. Cell Cycle. 2009; 8:567-572. [PubMed: 19197153]

26. Wander SA, Hennessy BT, Slingerland JM. Next-generation mTOR inhibitors in clinical oncology: how pathway complexity informs therapeutic strategy. J Clin Invest. 2011; 121:1231-1241. [PubMed: 21490404]

27. Lovejoy CA, Cortez D. Common mechanisms of PIKK regulation. DNA Repair (Amst). 2009; 8:1004-1008. [PubMed: 19464237]

28. Bosotti R, Isacchi A, Sonnhammer EL. FAT: a novel domain in PIK-related kinases. Trends Biochem Sci. 2000; 25:225-227. [PubMed: 10782091]

29. Walker EH, Perisic O, Ried C, Stephens L, Williams RL. Structural insights into phosphoinositide 3-kinase catalysis and signalling. Nature. 1999; 402:313-320. [PubMed: 10580505]

30. Sibanda BL, Chirgadze DY, Blundell TL. Crystal structure of DNA-PKcs reveals a large open-ring cradle comprised of HEAT repeats. Nature. 2010; 463:118-121. [PubMed: 20023628]

31. Nolen B, Taylor S, Ghosh G. Regulation of protein kinases; controlling activity through activation segment conformation. Mol Cell. 2004; 15:661-675. [PubMed: 15350212]

32. Miller S, et al. Shaping development of autophagy inhibitors with the structure of the lipid kinase Vps34. Science. 2010; 327:1638-1642. [PubMed: 20339072]

33. McMahon LP, Choi KM, Lin TA, Abraham RT, Lawrence JC Jr. The rapamycin-binding domain governs substrate selectivity by the mammalian target of rapamycin. Mol Cell Biol. 2002; 22:7428-7438. [PubMed: 12370290]

34. Sekulic A, et al. A direct linkage between the phosphoinositide 3-kinase-AKT signaling pathway and the mammalian target of rapamycin in mitogen-stimulated and transformed cells. Cancer Res. 2000; 60:3504-3513. [PubMed: 10910062]

35. Edinger AL, Thompson CB. An activated mTOR mutant supports growth factor-independent, nutrient-dependent cell survival. Oncogene. 2004; 23:5654-5663. [PubMed: 15133498]

36. Bao ZQ, Jacobsen DM, Young MA. Briefly bound to activate: transient binding of a second catalytic magnesium activates the structure and dynamics of CDK2 kinase for catalysis. Structure. 2011; 19:675-690. [PubMed: 21565702]

37. Madhusudan, Akamine P, Xuong NH, Taylor SS. Crystal structure of a transition state mimic of the catalytic subunit of cAMP-dependent protein kinase. Nat Struct Biol. 2002; 9:273-277. [PubMed: 11896404]

38. Brown EJ, et al. Control of p70 s6 kinase by kinase activity of FRAP in vivo. Nature. 1995; 377:441-446. [PubMed: 7566123]

39. Hsu PP, et al. The mTOR-regulated phosphoproteome reveals a mechanism of mTORC1-mediated inhibition of growth factor signaling. Science. 2011; 332:1317-1322. [PubMed: 21659604] 
40. Vilella-Bach M, Nuzzi P, Fang Y, Chen J. The FKBP12-rapamycin-binding domain is required for FKBP12-rapamycin-associated protein kinase activity and G1 progression. J Biol Chem. 1999; 274:4266-4272. [PubMed: 9933627]

41. Shor B, et al. A new pharmacologic action of CCI-779 involves FKBP12-independent inhibition of mTOR kinase activity and profound repression of global protein synthesis. Cancer Res. 2008; 68:2934-2943. [PubMed: 18413763]

42. Rodriguez A, et al. A conserved docking surface on calcineurin mediates interaction with substrates and immunosuppressants. Mol Cell. 2009; 33:616-626. [PubMed: 19285944]

43. Ohne Y, et al. Isolation of hyperactive mutants of mammalian target of rapamycin. J Biol Chem. 2008; 283:31861-31870. [PubMed: 18812319]

44. Reinke A, Chen JC, Aronova S, Powers T. Caffeine targets TOR complex I and provides evidence for a regulatory link between the FRB and kinase domains of Tor1p. J Biol Chem. 2006; 281:31616-31626. [PubMed: 16923813]

45. Urano J, et al. Point mutations in TOR confer Rheb-independent growth in fission yeast and nutrient-independent mammalian TOR signaling in mammalian cells. Proc Natl Acad Sci U S A. 2007; 104:3514-3519. [PubMed: 17360675]

46. Liu Q, et al. Discovery of 9-(6-aminopyridin-3-yl)-1-(3-(trifluoromethyl)phenyl)benzo[h] $[1,6]$ naphthyridin-2 $(1 \mathrm{H})$-one (Torin2) as a potent, selective, and orally available mammalian target of rapamycin (mTOR) inhibitor for treatment of cancer. J Med Chem. 2011; 54:1473-1480. [PubMed: 21322566]

47. Apsel B, et al. Targeted polypharmacology: discovery of dual inhibitors of tyrosine and phosphoinositide kinases. Nat Chem Biol. 2008; 4:691-699. [PubMed: 18849971]

48. Knight ZA, et al. A pharmacological map of the PI3-K family defines a role for p110alpha in insulin signaling. Cell. 2006; 125:733-747. [PubMed: 16647110]

49. Otwinowski Z, Minor W. Processing of X-ray Diffraction Data Collected in Oscillation Mode. Methods in Enzymol. 1997; 276:307-326.

50. Bricogne G, Vonrhein C, Flensburg C, Schiltz M, Paciorek W. Generation, representation and flow of phase information in structure determination: recent developments in and around SHARP 2.0. Acta Cryst D. 2003; 59

51. The CCP4 suite: programs for protein crystallography. Acta crystallographica. 1994; 50:760-763.

52. Jones TA, Zou JY, Cowan SW, Kjeldgaard M. Improved methods for building protein models in electron density maps and the location of errors in these models. Acta crystallographica. 1991; 47(Pt 2):110-119. [PubMed: 2025413]

53. Adams PD, et al. PHENIX: a comprehensive Python-based system for macromolecular structure solution. Acta Crystallogr D Biol Crystallogr. 2010; 66:213-221. [PubMed: 20124702] 


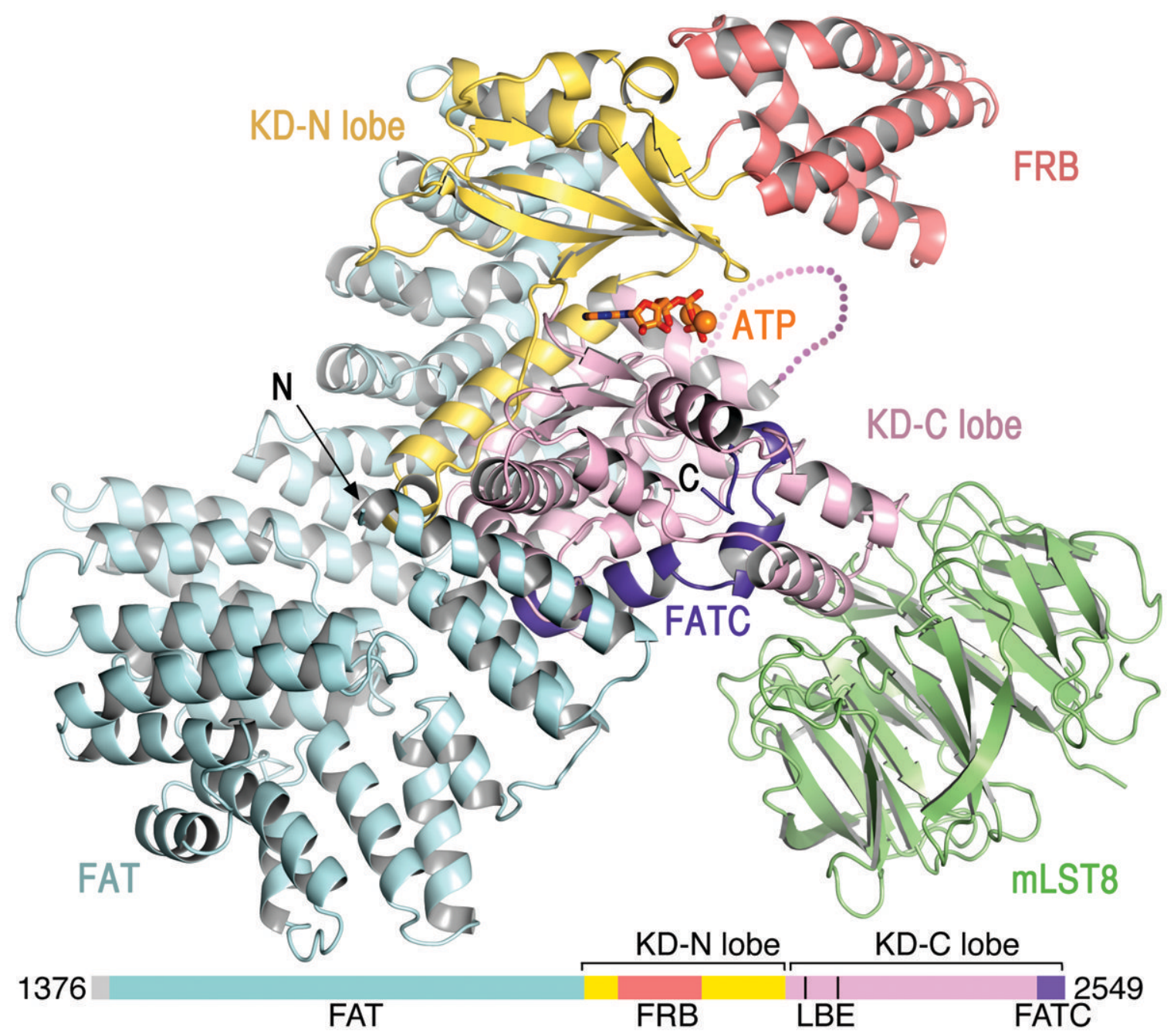

Figure 1. Structure of the $\mathrm{mTOR}^{\Delta \mathrm{N}_{-}}$-mLST8-ATP $\gamma \mathrm{S}-\mathrm{Mg}$ complex

mTOR is coloured as indicated in the linear schematic, mLST8 is coloured green, ATP is shown as sticks, and $\mathrm{Mg}^{2+}$ ions as spheres. 
a
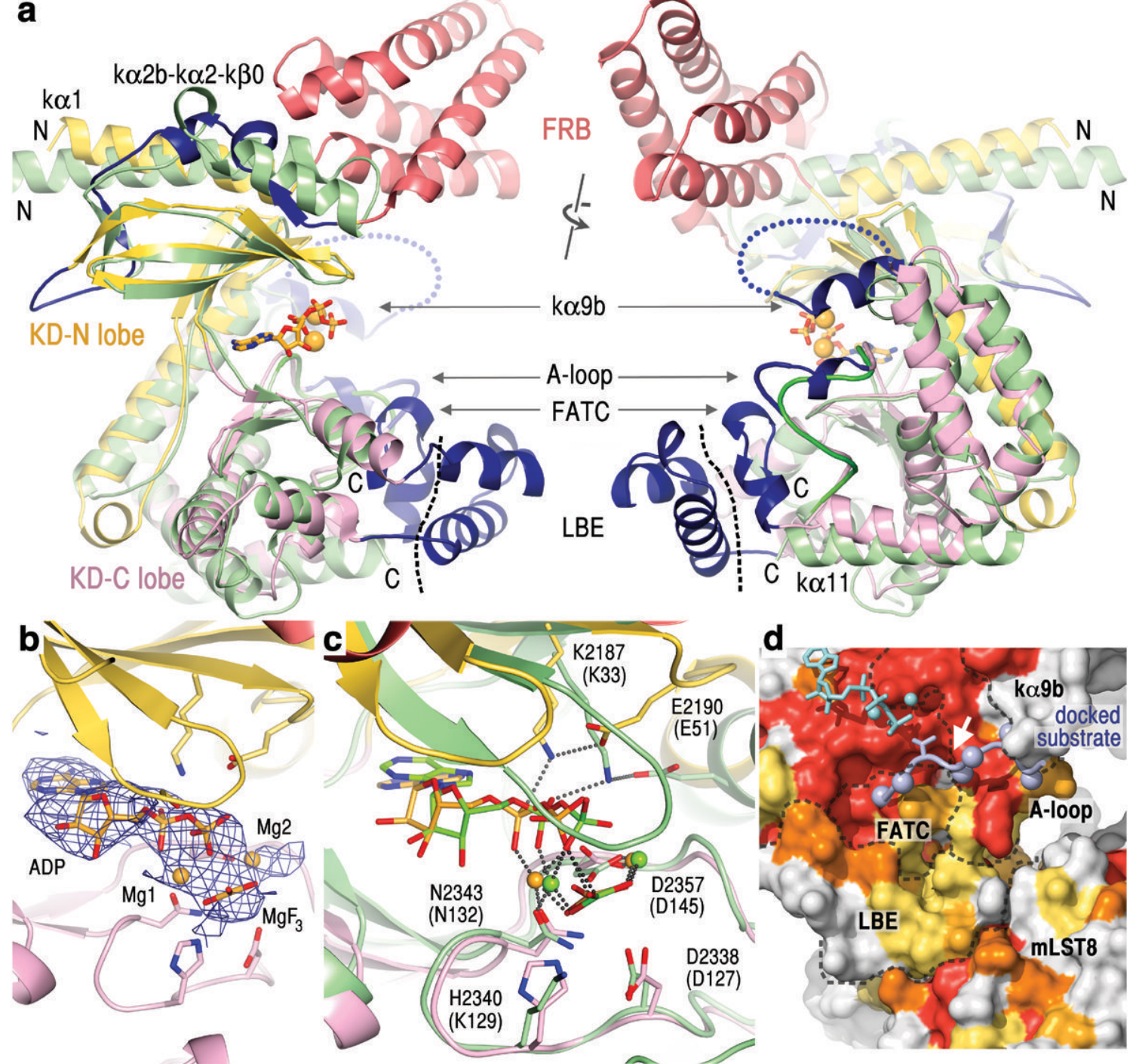

Figure 2. mTOR kinase domain and active site conformation

a, Superposition of the kinase domains of mTOR and PIK3C3 (PDB 3IHY; green) in two views rotated by $\sim 180^{\circ}$. Left view is related to that of Figure 1 . The FRB is colored red, and the remaining mTOR insertions dark blue. Black dashed line delineates the LBE, and the blue dotted loop indicates the disordered region between ka9b and ka10.

b, The $3.5 \AA F o-F c$ electron density, contoured at $2.5 \sigma$, of the mTOR TS complex before $\mathrm{ADP}-\mathrm{MgF}_{3}-\mathrm{Mg}_{2}$ was built.

c, Superposition of the mTOR and CDK2 TS (PDB 3QHW) complexes. CDK2 and its nucleotide are coloured green (its residue labels in parentheses).

d, Molecular surface representation of the C lobe portion of the mTOR catalytic cleft, coloured according to conservation entropy (red invariant in 22 orthologs, orange in 21, yellow-orange in 20, and yellow in 18-19). Dashed lines delineate the boundaries of structural elements (labelled). The docked substrate peptide is coloured light blue, with its threonine phosphorylation site shown as sticks, the remaining side chains as spheres $(\mathrm{C} \beta$ atoms), and its +1 position indicated by a white arrow. 

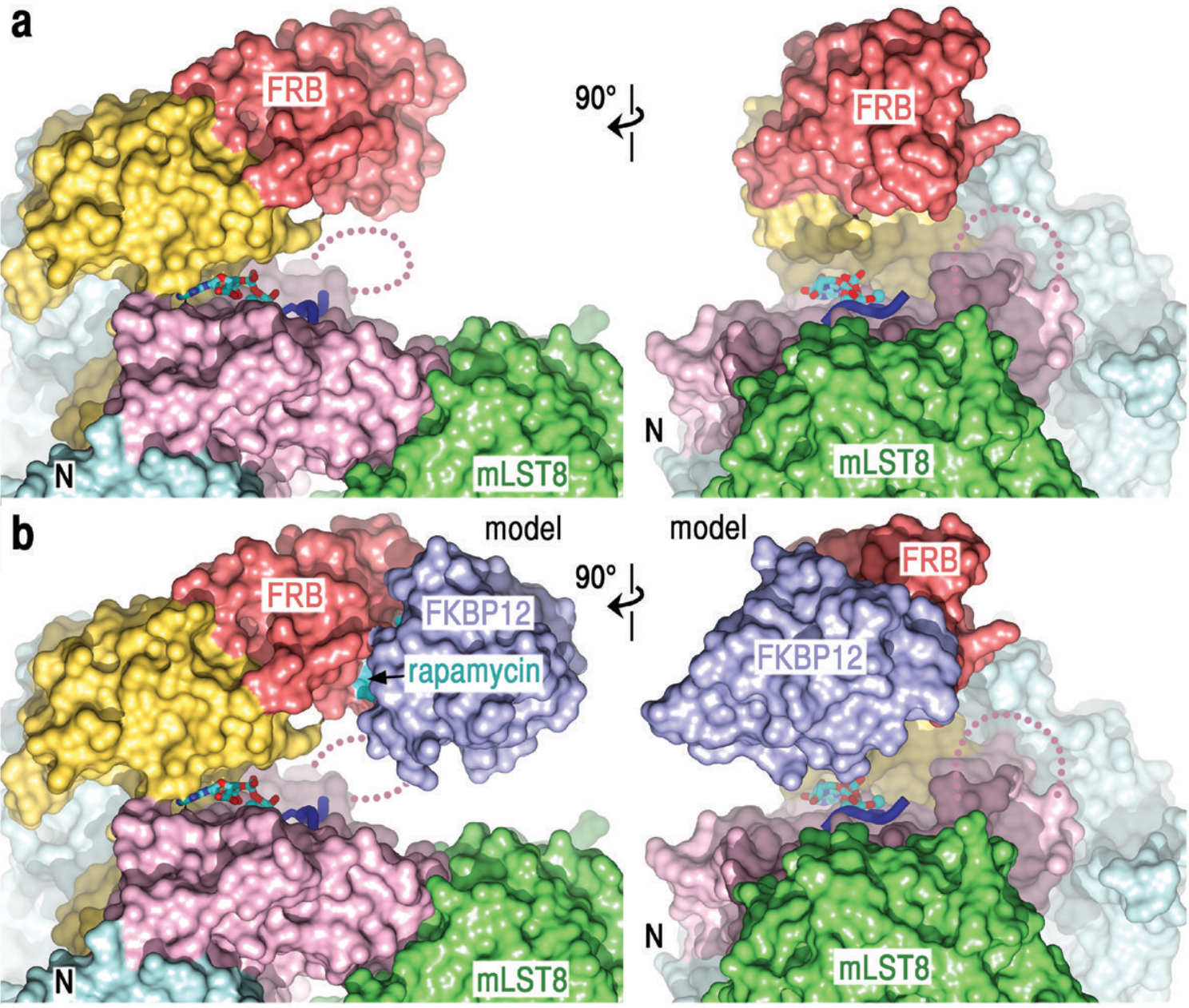

Figure 3. The kinase active site is recessed at the bottom of a deep cleft

a, Surface representation of $\mathrm{mTOR}^{\Delta \mathrm{N}}-\mathrm{mLST}$ - $\mathrm{ADP}-\mathrm{MgF}_{3}-\mathrm{Mg}_{2}$ in two orthogonal views.

Dark blue ribbon indicates the docked substrate peptide (positions -2 to +2 ) from Figure $2 \mathrm{~d}$.

b, Surface representation of the mTOR ${ }^{\Delta \mathrm{N}}$-mLST8-rapamycin-FKBP12 model. Rapamycin (cyan) and FKBP12 (blue) are labelled. 

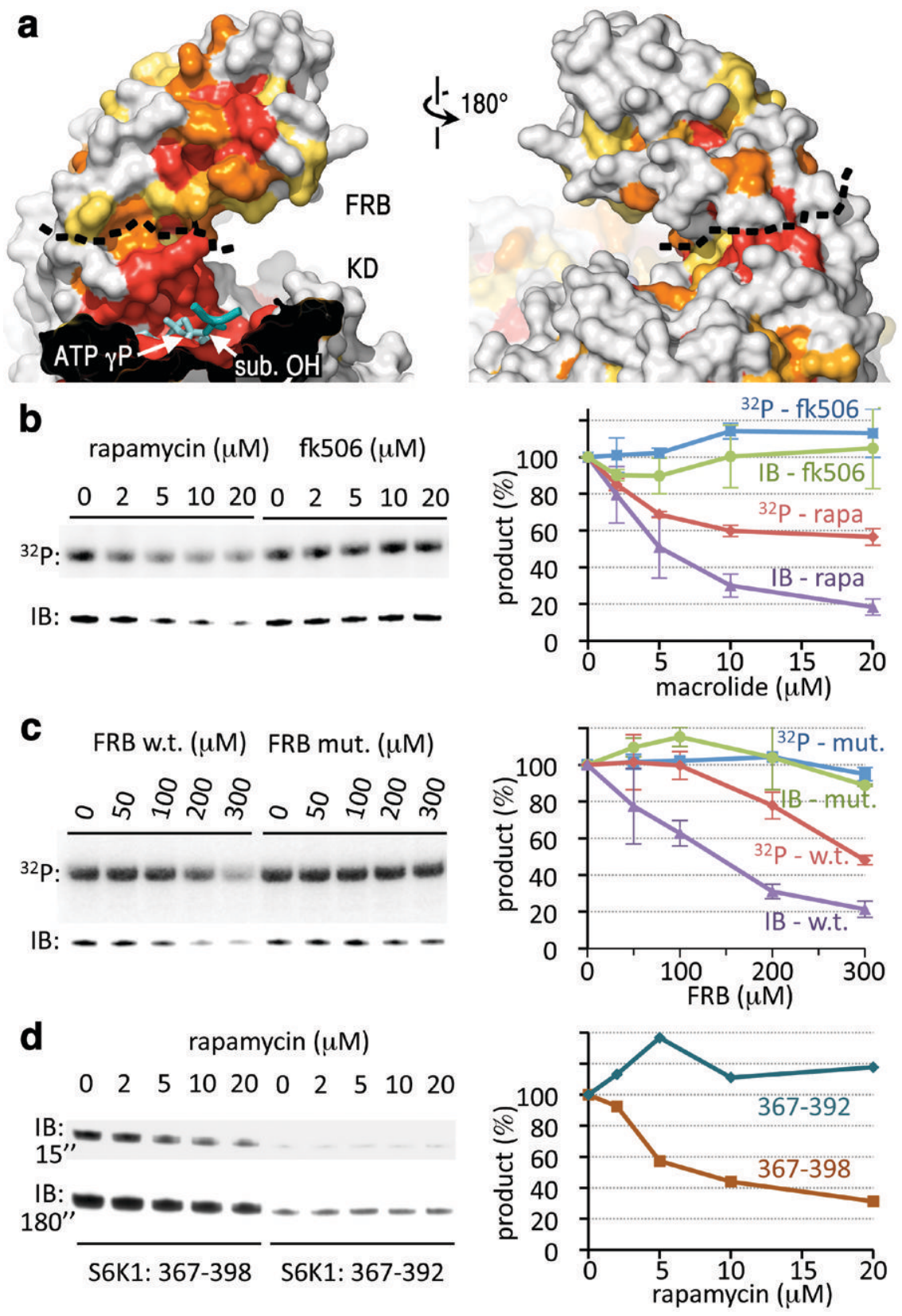

Figure 4. The rapamycin-binding site of the FRB recruits S6K1 into the catalytic cleft a, Surface representations of the FRB and portion of the catalytic cleft, coloured by sequence conservation as in Figure $2 \mathrm{~d}$. $\mathrm{ADP}-\mathrm{MgF}_{3}-\mathrm{Mg}_{2}$ is in cyan with the TS mimic $\mathrm{MgF}_{3}$ group labelled “ATP $\gamma \mathrm{P}$ ”. The black dashed line delineates the FRB-KD boundary. The docked substrate peptide from Figure $2 \mathrm{~d}$ is shown in blue (positions -1 to +1 ), with its phosphorylation site labelled "sub. OH".

b, Phosphorylation of S6K1 ${ }^{\mathrm{ki}}(10 \mu \mathrm{M})$ by mTOR ${ }^{\Delta \mathrm{N}}-\mathrm{mLST} 8(20 \mathrm{nM})$, measured by ${ }^{32} \mathrm{P}$ incorporation (top panel) and by immunoblotting with a phosphoThr389-specific antibody (lower panel), in the presence of the indicated concentrations of rapamycin or FK506. The 
average and standard deviation of three independent repetitions is plotted as a percentage of the $0 \mu \mathrm{M}$ macrolide reaction of each set.

c, Phosphorylation of S6K1 $1^{\mathrm{ki}}(10 \mu \mathrm{M})$ by $\mathrm{mTOR}^{\Delta \mathrm{N}}-\mathrm{mLST} 8(20 \mathrm{nM})$ in the presence of the indicated concentrations of the wild type or S2035I mutant FRB. Reactions were repeated twice, and results plotted as in (b).

d, Phosphorylation of GST-tagged S6K1 $1^{367-398}(10 \mu \mathrm{M})$ and S6K1 ${ }^{367-392}(10 \mu \mathrm{M})$ by $\mathrm{mTOR}^{\Delta \mathrm{N}}$-mLST8 (20 nM) in the presence of the indicated concentrations of rapamycin. Two different exposures (15 and $180 \mathrm{sec}$.) of the phosphoThr389-specific immunoblot are shown. The quantitation of the $15 \mathrm{sec}$. immunoblot is plotted on the right. 


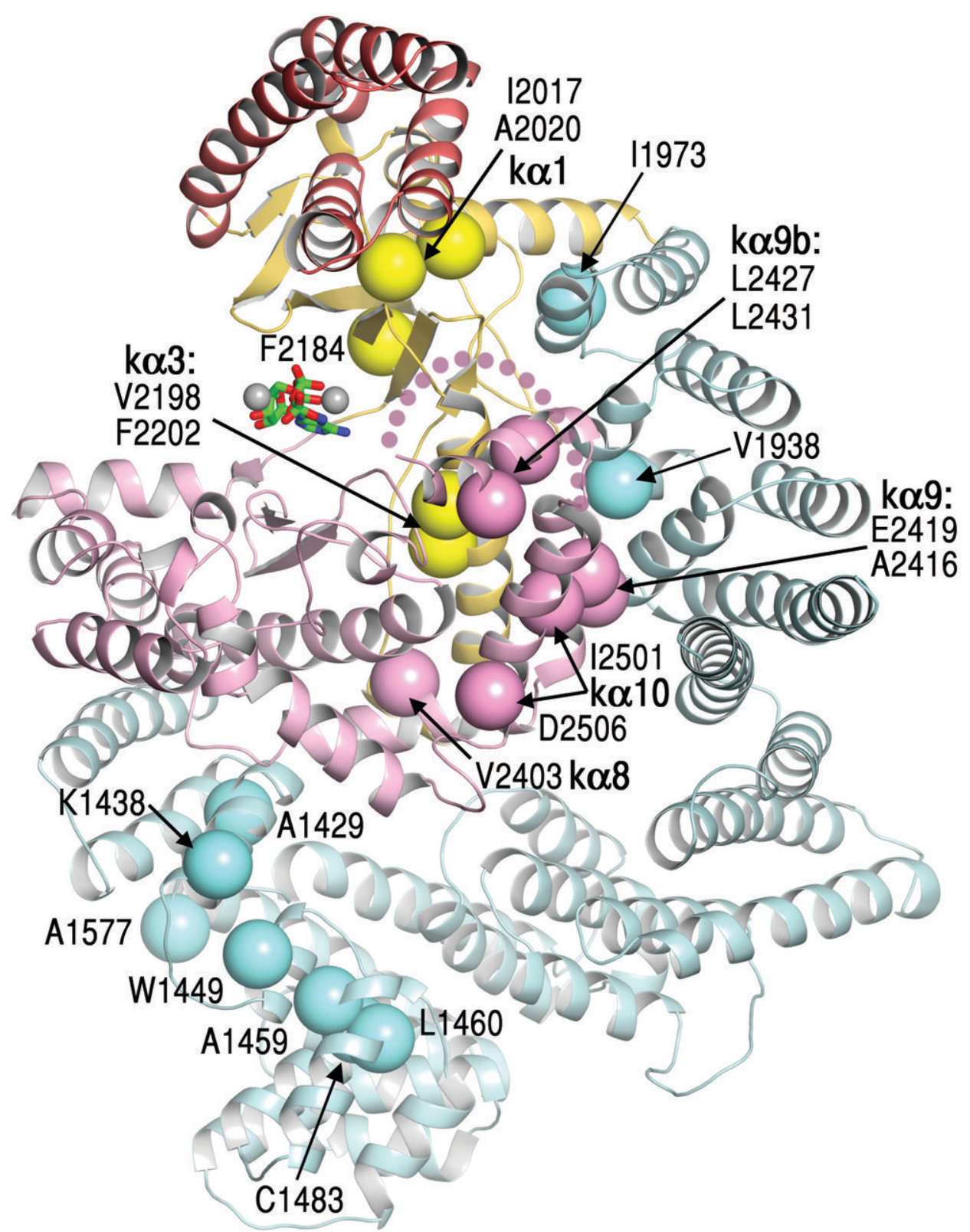

Figure 5. mTOR activating mutations map to structural elements involved in restricting active site access

Activating mutations ${ }^{43-45}$ reported for mTOR, yeast Tor 2 and Tor $1 \mathrm{p}$ are shown as large spheres (labelled, with structural elements in bold), coloured as in Figure 1. 


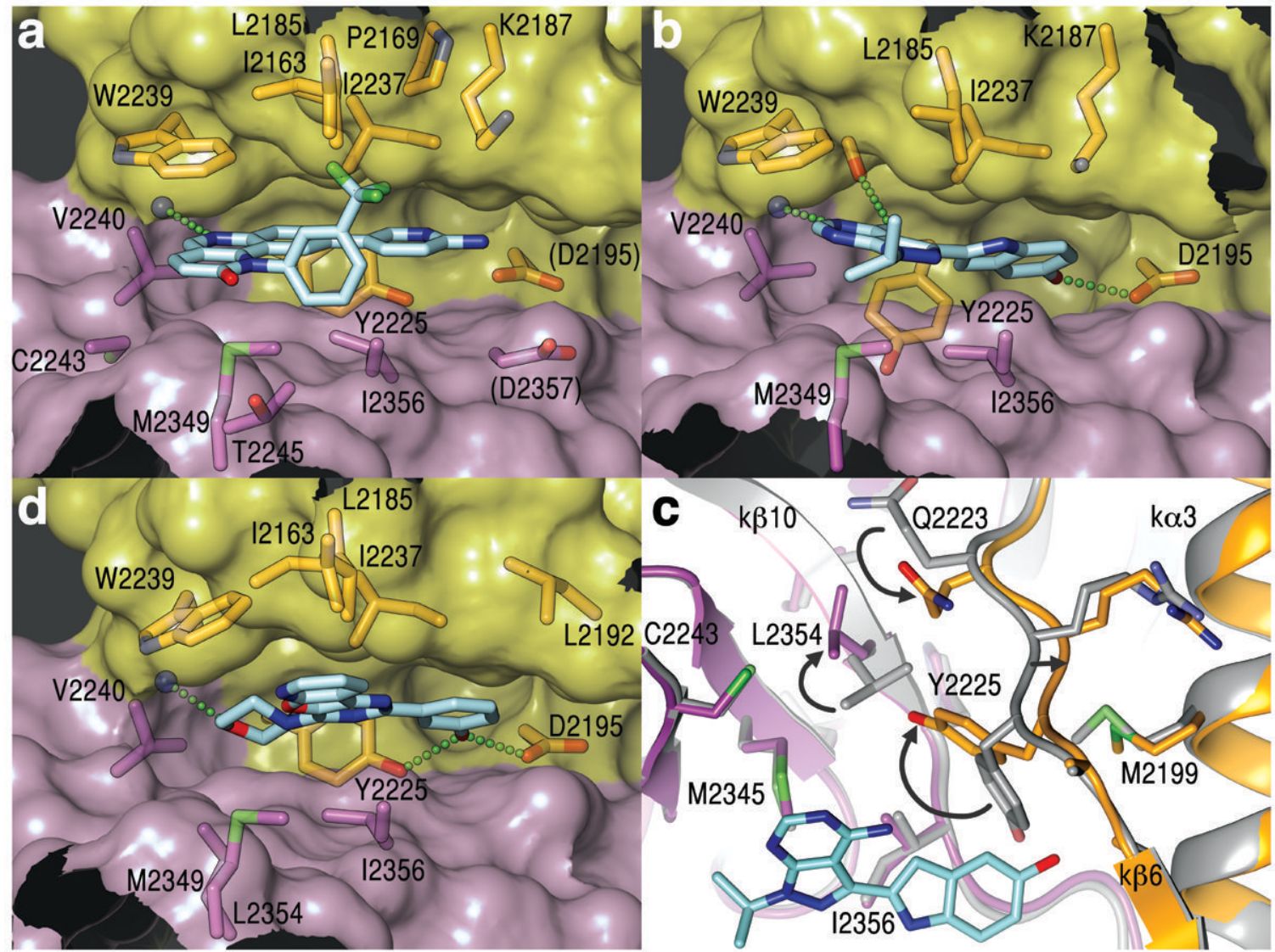

Figure 6. Structures of the Torin2, PP242 and PI-103 inhibitors bound to the mTOR catalytic cleft

a Stick representation of Torin2 (C atoms coloured cyan, $\mathrm{N}$ blue, $\mathrm{O}$ red, $\mathrm{F}$ green) and of mTOR residues within $4 \AA$ (except for D2195 and D2357). The mTOR cleft is shown in transparent surface representation, with the $\mathrm{N}$ lobe in yellow and $\mathrm{C}$ lobe in pink. Green dotted line indicates atoms within hydrogen-bonding distance and geometry.

b, PP242-mTOR structure, represented as in (a).

c, Conformational change in the inner hydrophobic pocket of mTOR on PP242 (cyan) binding. Arrows indicate side chain rotations and main chain shifts compared to ATP $\gamma \mathrm{S}$ bound mTOR (gray). View looking down the vertical axis of (b).

d, PI103-mTOR structure, represented as in (a). 